\title{
Phase-Field Simulation of Austenite to Ferrite Transformation and Widmanstätten Ferrite Formation in Fe-C Alloy
}

\author{
Akinori Yamanaka ${ }^{1, *}$, Tomohiro Takaki ${ }^{2}$ and Yoshihiro Tomita ${ }^{1}$ \\ ${ }^{1}$ Graduate School of Science and Technology, Kobe University, Kobe, 657-8501, Japan \\ ${ }^{2}$ Faculty of Maritime Sciences, Kobe University, Kobe, 658-0022, Japan
}

\begin{abstract}
The formation process of Widmanstätten ferrite plates during the isothermal austenite to ferrite transformation in Fe-C alloy is simulated by the phase-field method. The effects of the anisotropy of interfacial properties on the growth kinetics of Widmanstätten ferrite plates are investigated by the regularized gradient energy coefficient method, which enables us to introduce a wide range of interface anisotropy. It is found that by employing this method, a very sharp tip of the plate can be simulated and the morphology of Widmanstätten ferrite plate is in good agreement with the experimentally observed one. The simulation results of the growth of a single Widmanstätten ferrite plate suggest that the lengthening rate of the Widmanstätten ferrite plate increases with increasing strength of anisotropy, which causes the increase of interfacial energy at the tip. Furthermore, the simulations of the morphological changes of Widmanstätten ferrite from a grain boundary allotriomorph ferrite are performed. The results clarify that the growth of Widmanstätten ferrite plates from allotriomorph ferrite requires high anisotropy of interfacial energy. It is also proved that, in the early stage of the growth, the plate tips directly formed at the convex part of allotriomorph ferrite can preferentially develop into Widmanstätten ferrite plates due to the morphological instability. The distribution of Widmanstätten ferrite plates depends on the initial interface shape of the grain boundary allotriomorph ferrite. [doi:10.2320/matertrans.47.2725]
\end{abstract}

(Received May 17, 2006; Accepted July 3, 2006; Published November 15, 2006)

Keywords: phase-field method, Widmanstätten ferrite, interface anisotropy, iron-carbon alloy, phase transformation

\section{Introduction}

The mechanical properties of steels are closely related to the morphology and composition of microstructures produced during the austenite to ferrite transformation. Therefore, it is essential for the improvement of the properties of steel to understand the transformation kinetics and to predict the evolution of microstructures. In particular, the morphological change from grain boundary allotriomorph ferrite to ferrite sideplates, that is, Widmanstätten ferrite, is often observed at temperatures between Ae 3 and Ae1, and the formation kinetics of Widmanstätten ferrite have been the subject of numerous studies.

However, it is difficult to investigate the evolution of the microstructures during the transformation by only experiments, since the austenite to ferrite transformation involves a complex competition between interface migration and fast solute diffusion. Therefore, the mechanism of Widmanstätten ferrite formation has been controversially as either diffusional or displacive growth. ${ }^{1-4)}$ From the view point of the diffusional growth model, some solutions based on thermodynamic calculations, such as the Ivantsov model, ${ }^{5)}$ ZenerHillert model ${ }^{6)}$ and Trivedi model, ${ }^{7)}$ have been proposed for the lengthening rate of Widmanstätten ferrite plates. These models take into consideration of the effect of capillarity of the plate tip and provide good agreement with the experimental lengthening rate of Widmanstätten ferrite. However, the complex microstructural evolutions cannot be predicted using those models in two or three dimensions.

Recently, a phase-field method ${ }^{8,9)}$ is proposed to simulate the complicated microstructure evolution, for example, dendric solidification of pure materials and alloys, grain growth of polycrystals and martensitic transformation. ${ }^{10-12)}$ In this method, introducing an order parameter, i.e., phase

*Graduate Student, Kobe University field $\phi$, enables us to consider the interface region as a continuous change of $\phi$ and to remove the necessity of tracking the moving interface. Therefore, the microstrucure evolutions which include interface migration, temperature and composition changes can be simulated by solving a set of governing equations.

Loginova et al. proposed a phase-field model of the austenite to ferrite transformation in $\mathrm{Fe}-\mathrm{C}$ alloy ${ }^{13)}$ and developed their model to simulate the formation of Widmanstätten ferrite plate. ${ }^{14)}$ They successfully simulated the morphological change of the ferrite to Widmanstätten plate and investigated the evolution of Widmanstätten structure in terms of the anisotropy of interfacial properties. However, as they discussed in their paper, the simulated tips of the plate is not sharp as shown in experimentally observed one.

In the present analysis, we extend the phase-field model proposed by Loginova et al. ${ }^{14)}$ to simulate realistic morphology of Widmanstätten ferrite plate by employing the regularized gradient energy coefficient method, which enables us to introduce a wide range of interface anisotropy. We first evaluate the effects of the anisotropy of the interfacial energy, which depends on the coherence of the interface, the growth process and the morphology of the Widmanstätten plate. Then, the formation of Widmanstätten ferrite from allotriomorph ferrite with perturbated interface morphology is simulated to study the growth kinetics and morphological change of microstructures.

\section{Phase-Field Model}

\subsection{Governing equations}

The total Gibbs free energy of Fe-C alloy is postulated to be the form of the Ginzburg-Landau-type Gibbs free energy functional defined as the sum of chemical free energy and gradient free energy, ${ }^{13)}$ 


$$
G=\int_{V}\left[g\left(\phi, u_{c}, T\right)+\frac{\epsilon(\theta)^{2}}{2}|\nabla \phi|^{2}\right] d V
$$

where $\phi$ is the phase field which varies smoothly across the interface from $\phi=1$ in the ferrite phase to $\phi=0$ in the austenite phase, and $u_{c}$ is the carbon concentration which is related to the normal mole fraction of carbon $x_{C}$ as $u_{c}=$ $a x_{C} /\left\{c\left(1-x_{C}\right)\right\} . a$ and $c$ are the site numbers of iron and carbon atoms, respectively, which are defined as $a=c=1$ in the austenite phase and $a=1$ and $c=3$ in the ferrite phase. $T$ is the absolute temperature. $\epsilon(\theta)$ and $\theta$ are the gradient energy coefficient and the orientation angle, respectively, which are discussed later in detail.

The chemical free energy density of Fe-C alloy $g\left(\phi, u_{c}, T\right)$ is defined by the chemical free energy of pure ferrite $g^{\alpha}\left(u_{c}, T\right)$ and that of pure austenite $g^{\gamma}\left(u_{c}, T\right)$ as the following function:

$$
\begin{aligned}
g\left(\phi, u_{c}, T\right)= & p(\phi) g^{\alpha}\left(u_{c}, T\right) \\
& +(1-p(\phi)) g^{\gamma}\left(u_{c}, T\right)+W q(\phi) .
\end{aligned}
$$

Here, $p(\phi)=\phi^{3}\left(10-15 \phi+6 \phi^{2}\right)$ is the energy density function. $q(\phi)=\phi^{2}(1-\phi)^{2}$ is the double-well potential function, and $W$ is the energy barrier height of the doublewell potential, which is related to the interfacial energy $\sigma$ and interfacial thickness $\delta$ as $W=6 \sigma b / \delta$. We assume that the interface region is $\lambda \leq \phi \leq 1-\lambda$ and $b=\tanh ^{-1}(1-2 \lambda)$, where $\lambda$ is set to be 0.1 .15 )

The governing equations of the phase field and carbon concentration are derived by assuming that the total free energy decreases monotonically with time. The equation governing the evolution of a nonconserved order parameter, phase field $\phi$, is derived by the Allen-Cahn equation ${ }^{16)}$ as follows:

$$
\begin{aligned}
\frac{\partial \phi}{\partial t}= & -M_{\phi} \frac{\delta G}{\delta \phi} \\
= & M_{\phi}\left\{\nabla \cdot\left(\epsilon(\theta)^{2} \nabla \phi\right)-4 W \phi(1-\phi)\right. \\
& \times\left(-\frac{15}{2 W} \phi(1-\phi) \Delta g^{\gamma \alpha}+\phi-\frac{1}{2}\right) \\
& \left.-\frac{\partial}{\partial x}\left(\epsilon(\theta) \frac{\partial \epsilon(\theta)}{\partial \theta} \frac{\partial \phi}{\partial y}\right)+\frac{\partial}{\partial y}\left(\epsilon(\theta) \frac{\partial \epsilon(\theta)}{\partial \theta} \frac{\partial \phi}{\partial x}\right)\right\},
\end{aligned}
$$

where $\Delta g^{\gamma \alpha}=g^{\gamma}\left(u_{c}, T\right)-g^{\alpha}\left(u_{c}, T\right)$ is the chemical driving force for the transformation. $M_{\phi}$ is the kinetic parameter related to the mobility of the ferrite-austenite interface $M,{ }^{17}$ ) the potential height $W$ and the mean value of the gradient coefficient $\epsilon_{0}$ :

$$
M_{\phi}=\frac{\sqrt{2 W}}{6 \epsilon_{0}} M=\frac{\sqrt{2 W}}{6 \epsilon_{0}} \cdot 0.035 \exp \left(\frac{-17700}{T}\right) .
$$

The governing equation of a conserved order parameter, carbon concentraion $u_{c}$, is derived by the Cahn-Hilliard nonlinear diffusion equation ${ }^{18)}$ as

$$
\begin{aligned}
\frac{\partial u_{c}}{\partial t} & =\nabla \cdot\left\{M_{c}\left(\phi, u_{c}, T\right) \nabla\left(\frac{\delta G}{\delta u_{c}}\right)\right\} \\
& =\nabla \cdot\left\{M_{c}\left(\phi, u_{c}, T\right)\left(\frac{\partial^{2} g}{\partial u_{c}^{2}} \nabla u_{c}+\frac{\partial^{2} g}{\partial u_{c} \partial \phi} \nabla \phi\right)\right\},
\end{aligned}
$$

where $M_{c}\left(\phi, u_{c}, T\right)$ is the diffusion coefficient of carbon atoms in $\mathrm{Fe}-\mathrm{C}$ alloy, and is related to physical values by the following equation: ${ }^{19)}$

$$
M_{c}\left(\phi, u_{c}, T\right)=u_{c} y_{v} v_{m} M_{c} .
$$

Here, $v_{m}$ is the molar volume of Fe-C alloy. $y_{v}$ is the vacant sublattice concentration postulated to have phase dependence with $y_{v}^{\alpha}=1-u_{c} / 3$ in the ferrite phase and $y_{v}^{\gamma}=1-u_{c}$ in the austenite phase, and we use

$$
\begin{aligned}
y_{v} & =p(\phi) y_{v}^{\alpha}+(1-p(\phi)) y_{v}^{\gamma} \\
& =p(\phi)\left(1-\frac{u_{c}}{3}\right)+(1-p(\phi))\left(1-u_{c}\right) .
\end{aligned}
$$

Since the diffusional mobility of carbon atom in each phase could differ by several orders of the magnitude, we assume a smooth change of the mobility of carbon atom $M_{c}$ inside the interface region by the following equation with the mobility of carbon atoms in the pure ferrite phase, $M_{c}^{\alpha}$, and the pure austenite phase, $M_{c}^{\gamma} \cdot{ }^{20,21)}$

$$
M_{c}=\left(M_{c}^{\alpha}\right)^{p(\phi)}\left(M_{c}^{\gamma}\right)^{(1-p(\phi))}
$$

\subsection{Interface anisotropy}

The interfacial energy varies depending on the crystallographic orientation difference between the product phase and the matrix. The orientation dependence of the interfacial energy causes the interface anisotropy that strongly influences the morphology of microstructures. In this model, we introduce the interface anisotropy as the anisotropy of the gradient energy coefficient $\epsilon(\theta)$ related to the interfacial energy $\sigma$ and interfacial thickness $\delta$ by the following equation:

$$
\epsilon(\theta)=\epsilon_{0} \eta(\theta)=\sqrt{\frac{3 \sigma \delta}{b}}\left\{1+\xi \cos k\left(\theta-\theta_{0}\right)\right\},
$$

where $\eta(\theta)$ is the anisotropy function, $\xi$ is the strength of anisotropy, $k$ is the mode number of anisotropy, $\theta_{0}$ is the preferred growth orientation and the orientation $\theta$ is defined as the angle between the interface normal and the $x$-axis, as indicated in Fig. 1(a). In this study, we introduce a wide range of interface anisotropy in order to study the formation of Widmanstätten ferrite. However, when we introduce high anisotropy, such as $\xi \geq 1 /\left(k^{2}-1\right)$, the equilibrium shape cannot be provided in the range of $2 \pi i / k-\theta_{m} \leq \theta-$ $\theta_{0} \leq 2 \pi i / k+\theta_{m}$, which corresponds to the part (A) indicated by arrows in Fig. 1(b). $\theta_{m}$ is the first missing orientation showed in Fig. 1(a) and $i$ denotes integers from 1 to $k-1$. In order to consider such high interface anisotropy and provide the equilibrium shape, we employ the following regularized gradient energy coefficient for missing orientations. ${ }^{22)}$

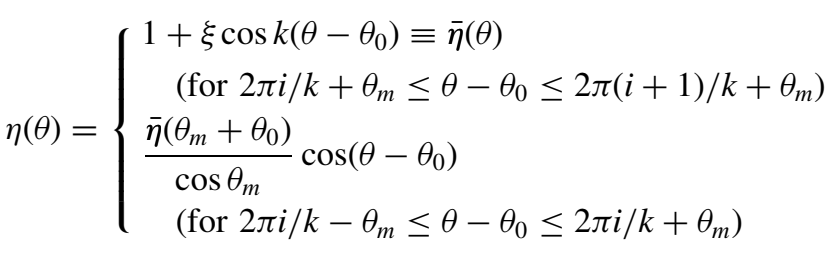

\section{Numerical Simulation}

The governing equations, eqs. (3) and (5), are solved as a 

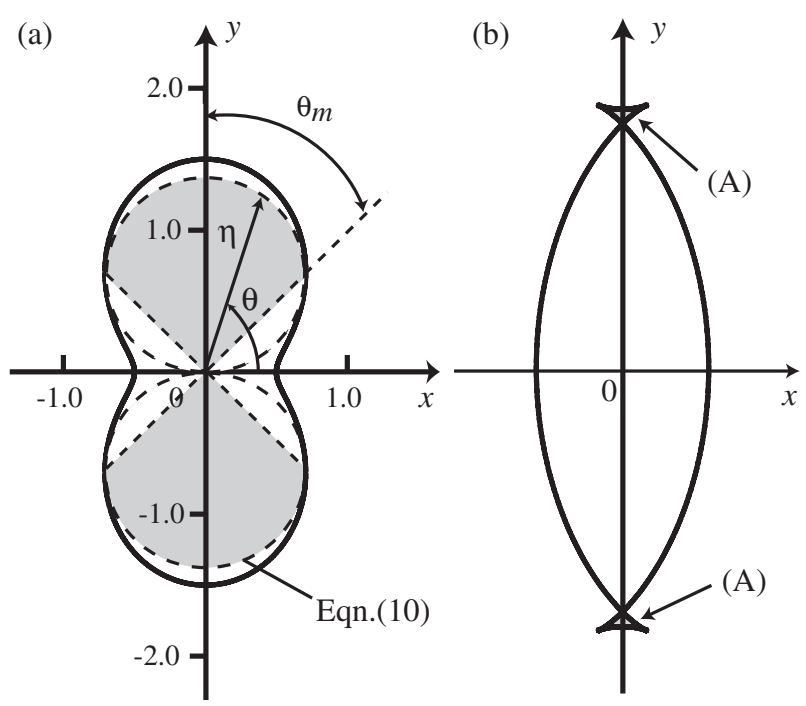

Fig. 1 Interface anisotropy for $k=2, \xi=0.6, \theta_{0}=90^{\circ}$. (a) Polar plot of anisotropy function $\eta(\theta)$ and (b) equilibrium shape of interface.

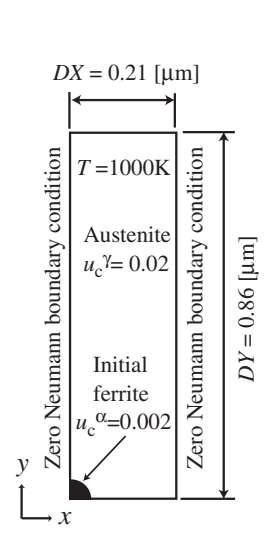

(a)

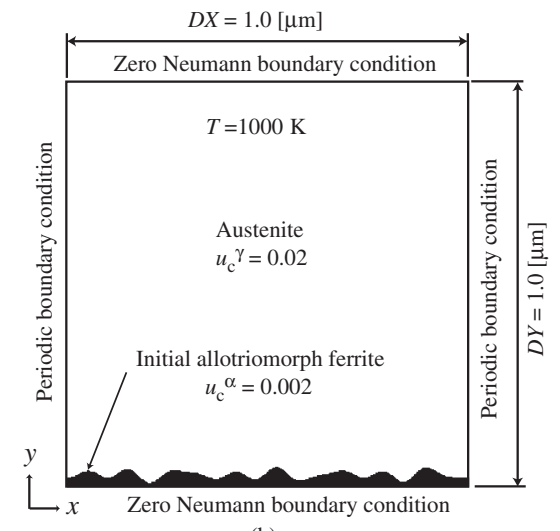

(b)
Fig. 2 Computational model, initial conditions and boundary conditions (a) for the simulation of the growth of a single Widmanstätten ferrite plate and (b) for the simulation of Widmanstätten ferrite plates formation from grain boundary allotriomorph ferrite.

two-dimensional problem by the Crank-Nicholson finite difference method for time and the adaptive finite element method with isoparametric quadrilateral elements for space. ${ }^{15)}$ The levels of adaptive mesh refinement are set to six, and the smallest mesh size is $d x_{5}=2^{-5} \times d x_{0}$, where $d x_{0}$ and $d x_{5}$ stand for the sizes of the level 0 mesh and the level 5 mesh, respectively. The mesh refinement is conducted when the average of four nodal values of the phase field, $\phi_{\text {ave }}$, and the average of $|\nabla c|$ at four Gaussian points in an element, $|\nabla c|_{\text {ave }}$, satisfy the conditions $0.01 \leq \phi_{\text {ave }} \leq 0.9$ or $|\nabla c|_{\text {ave }} \geq$ 0.3 . Other conditions lead to the coarsening of the adaptive meshes.

Figure 2(a) shows the computational model, initial conditions and boundary conditions for the growth of a single Widmanstätten ferrite plate, as discussed in section 5.1. The initial circular nucleus is set at the origin of the $D X \times$ $D Y=0.21 \times 0.86 \mu \mathrm{m}$ rectangular computational domain. Since the shape of Widmanstätten ferrite plate is assumed to be symmetric with respect to the growth direction, we deal with half of the plate by employing zero Neumann boundary conditions on both sides, and the preferred plate growth direction of $\theta_{0}=\pi / 2$ is adopted.

Figure 2(b) shows the computational model, initial conditions and boundary conditions for the numerical simulations of the formation of Widmanstätten ferrite plates from grain boundary allotriomorph ferrite, as discussed in section 5.2. The size of the square computational domain is $D X=$ $D Y=1.0 \mu \mathrm{m}$. In this model, the growth direction $\theta_{0}=$ $7 \pi / 18$ is adopted. We employ periodic boundary conditions at both sides and zero Neumann boundary conditions at upper and lower ends for both order parameters. The initial grain boundary allotriomorph ferrite is set at the bottom of the computational domain, as shown in Fig. 2(b). In order to study the effects of the initial interface morphology on the growth kinetics, the initial ferrite-austenite interface of allotriomorph ferrite is disturbed by a combination of sinusoidal curves. The height of the initial interface of allotriomorph ferrite along the $y$-axis, $h(x)$, is defined as the following function of coordinate $x$ :

$$
h(x)=h_{0}+\sum_{i=1}^{M} a_{i} \sin \left(\frac{2 \pi N_{i}}{D X} x+\psi_{i}\right),
$$

where $h_{0}, a_{i}, N_{i}$ and $\psi_{i}$ are the average height of the interface, the height of waves, the wave numbers on the $x$ axis and the initial phase of the sinusoidal wave, respectively. The index $i$ is the number of sinusoidal waves and is defined as $M=3$ in this study. We initially disturb the interface morphology with the following parameters: $h_{0}=33 \mathrm{~nm}, a_{1}=a_{3}=6.6 \mathrm{~nm}$, $a_{2}=9.9 \mathrm{~nm}, \psi_{1}=\pi / 2, \psi_{2}=0$ and $\psi_{3}=3 \pi / 5$.

In both simulation models, the transformation temperature is set to be constant at $T=1000 \mathrm{~K}$ due to isothermal transformation. Initial carbon concentrations are in equilibrium, $u_{c}^{\alpha}=0.002$, in the ferrite phase and supersaturated, $u_{c}^{\gamma}=0.02$, in the austenite phase. The following material and phase-field parameters are used: the mode number of anisotropy $k=2$, interface thickness $\delta=6 d x_{5}=20 \mathrm{~nm}$, interface energy $\sigma=1.0 \mathrm{~J} / \mathrm{m}^{2}$ and the molar volume of $\mathrm{Fe}$ C alloy $v_{m}=7.0 \times 10^{-6} \mathrm{~m}^{3} / \mathrm{mol}$.

\section{Results and Discussion}

\subsection{Evolution of single Widmanstätten ferrite plate}

In order to study the effects of interfacial anisotropy on the growth process, the growth of a single Widmanstätten ferrite plate from a small circular ferrite nucleus in supersaturated austenite is simulated. In this section, we set the interfacial energy at the broad side of the plate to be constant at 0.25 $\mathrm{J} / \mathrm{m}^{2}$, which corresponds to the value for a semi-coherent interface. $^{23)}$

Figure 3 shows the morphologies of a single Widmanstätten ferrite plate and distributions of carbon concentration for various strength of anisotropy values, $\xi=0.3,0.4,0.5$ and 0.6 , where the length of all plates is $0.5 \mu \mathrm{m}$ and the computational domain is reflected about the $y$-axis. An initial circular nucleus changes its morphology to a platelike shape with parallel broad sides and a very sharp tip, due to the introduction of the high interface anisotropy. The morphology of the Widmanstätten ferrite plate simulated in the present study is in good agreement with the experimentally 

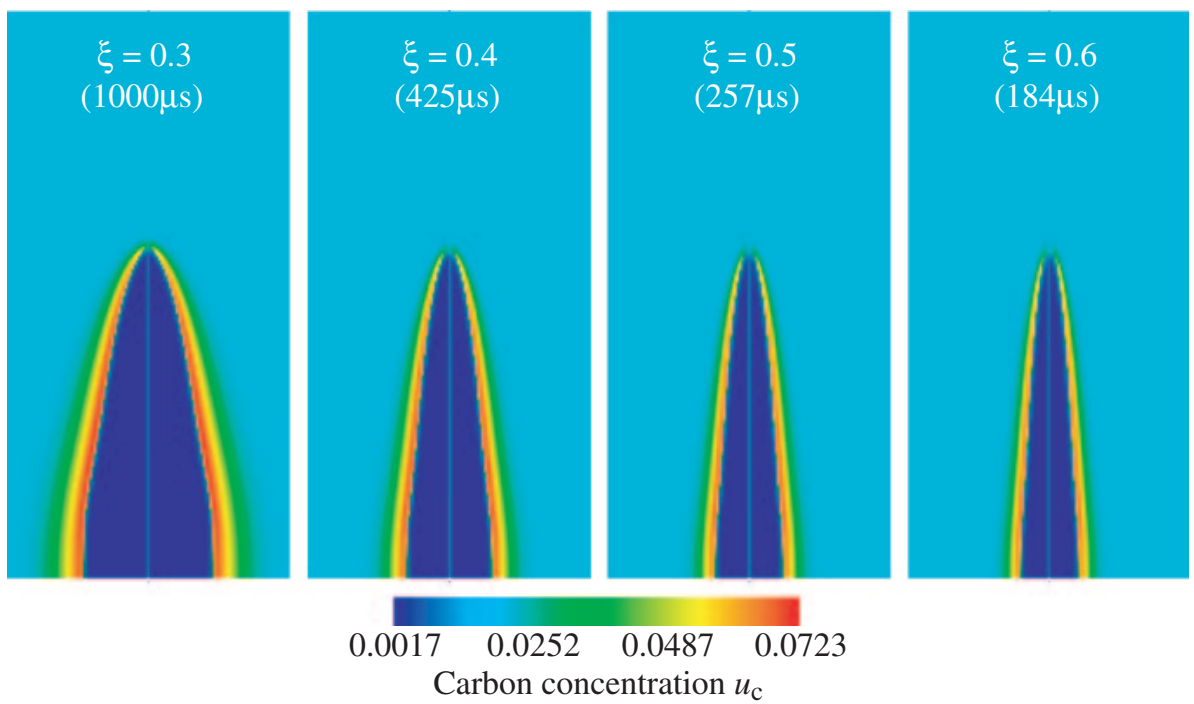

Carbon concentration $u_{\mathrm{c}}$

Fig. 3 Morphologies of a single Widmanstätten ferrite plate and distributions of carbon concentration for strength of anisotropy $\xi=0.3$, $0.4,0.5$ and 0.6. The times when the plate length reached $0.5 \mu \mathrm{m}$ are included in the figures.

observed plate shape. ${ }^{24)}$ The results clarify that a sharper tip and more realistic morphology of the plate than that obtained with a similar analysis ${ }^{14)}$ can be represented by employing the regularized gradient energy coefficient method.

The results show that the ferrite-austenite interface migrates with the buildup of carbon atoms in front of the interface, the amount of which varies around the plate, because of carbon diffusion from the newly formed ferrite into the austenite matrix during the austenite to ferrite transformation. As discussed in the literatures, ${ }^{25,26)}$ in view of the interaction between the diffusion field of carbon and the migration of the ledge at the interface, the present study also reveals that the distribution of the carbon diffusion field plays an important role in the formation of the platelike ferrite. That is, as shown in Fig. 3, the interface migration in the direction of plate thickness is greatly suppressed due to the large diffusion field of carbon, which provides the austenite phase with little carbon supersaturation, at the broad side of the plate. On the other hand, at the plate tip, the diffusion length of carbon becomes the shortest and the interface can migrate fast. Such behavior leads to the formation and lengthening of a platelike ferrite.

Figure 4 shows the carbon concentration profiles along the center of the plate. The carbon buildup in austenite in the vicinity of the interface becomes considerably small with increasing the strength of anisotropy. Hence, the lengthening rate of the plate shown in Fig. 5 increases with the increase of strength of anisotropy, which corresponds to the increase of interfacial energy at the tip. It is additionally noted that the carbon concentration at the interface does not obey the local equilibrium condition, as suggested in the previous studies. ${ }^{13,27)}$ In the case of the highest interface anisotropy, $\xi=0.6$, it is observed that the carbon buildup in front of the interface completely vanishes and a high lengthening rate is attained. From these results, we can conclude that the increase of the strength of anisotropy causes a high lengthening rate and sharper tip shapes of the Widmanstätten ferrite plate.
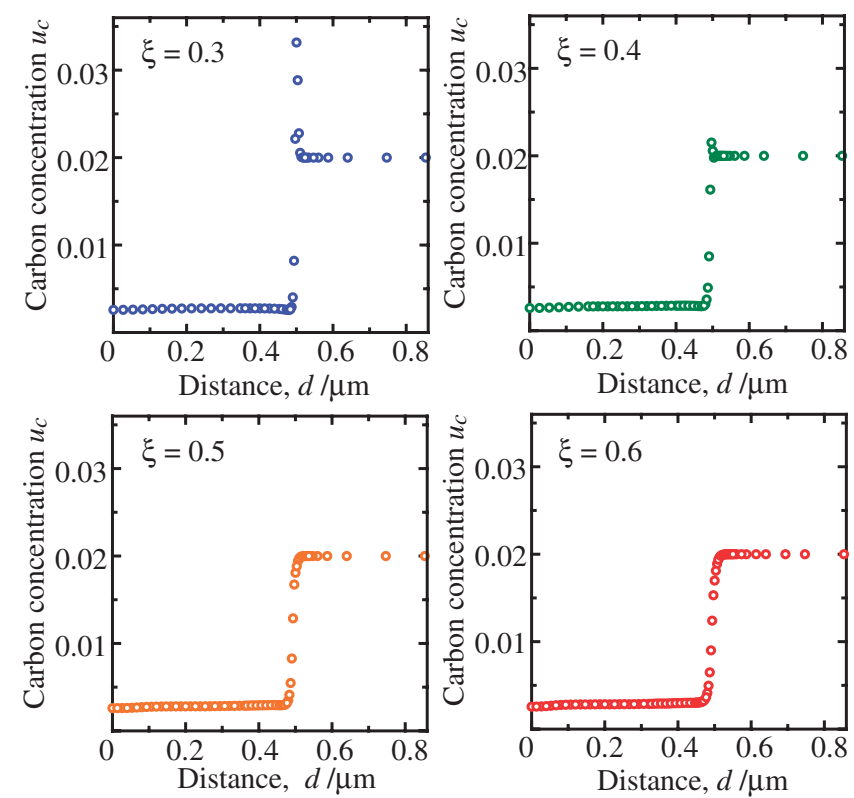

Fig. 4 Carbon concentration profiles along $y$-axis in Fig. 3 for strength of anisotropy $\xi=0.3,0.4,0.5$ and 0.6 .

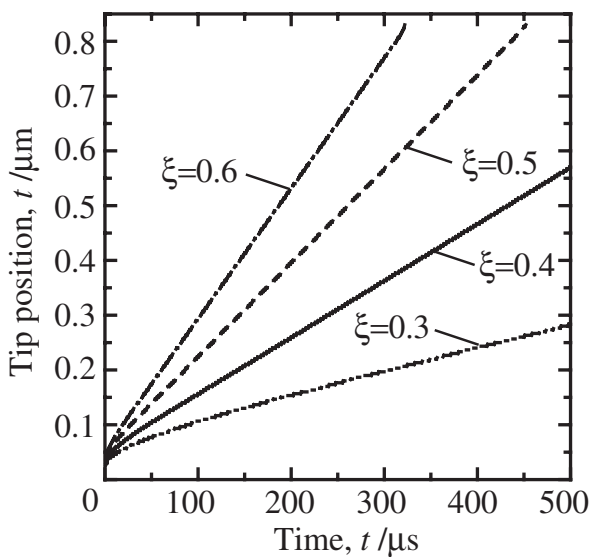

Fig. 5 The time changes of tip position of plates for strength of anisotropy $\xi=0.3,0.4,0.5$ and 0.6 . 

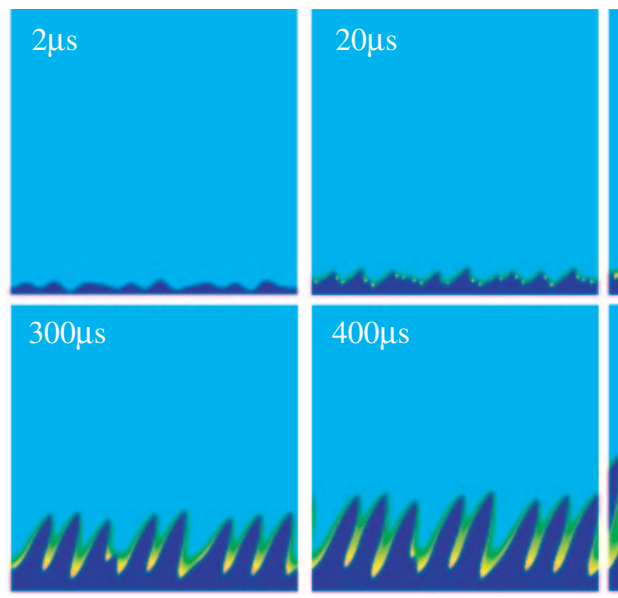

0.0014
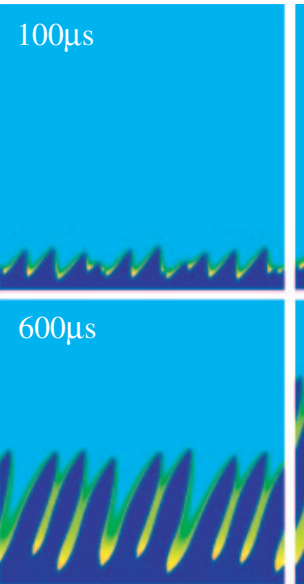

0306

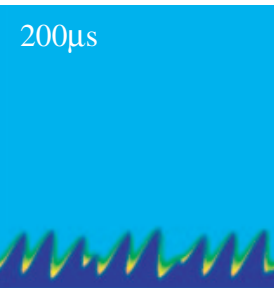

$1000 \mu \mathrm{s}$

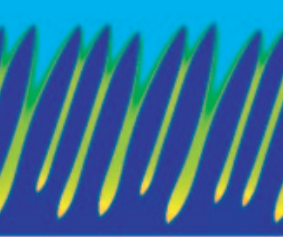

Carbon concentration $u_{\mathrm{c}}$

Fig. 6 Growth of Widmanstätten ferrite plates for $\xi=0.6$.
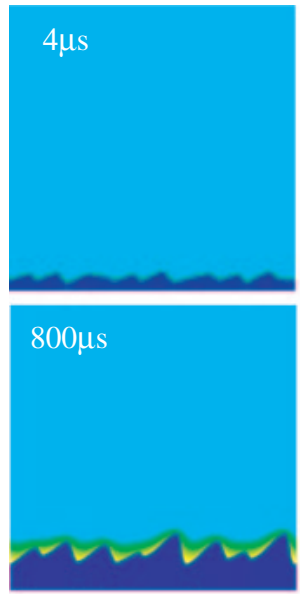
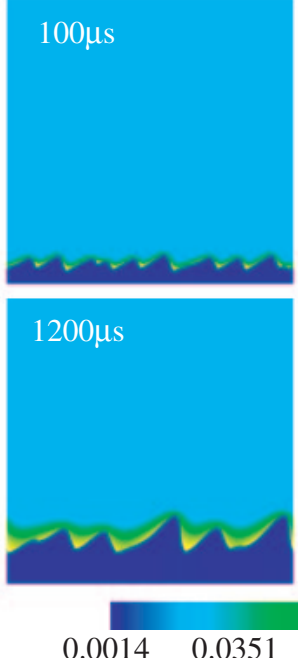

Carbon concentration $u_{\mathrm{c}}$
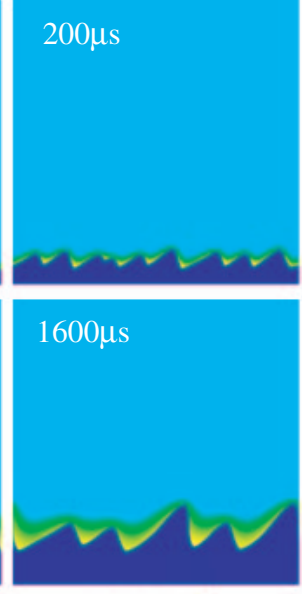
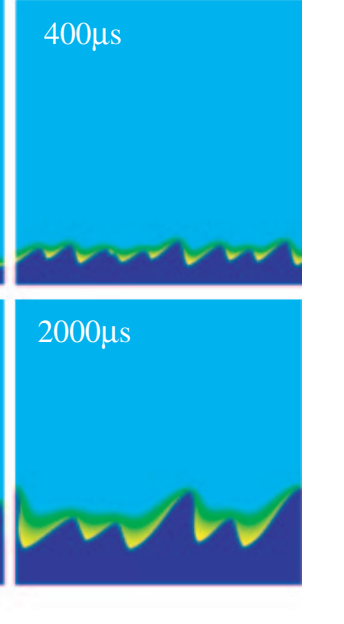

Fig. 7 Growth of Widmanstätten ferrite plates for $\xi=0.4$.

\subsection{Growth of Widmanstätten ferrite plates from allo- triomorph ferrite}

\subsubsection{Effects of strength of anisotropy}

The time sequence of the growth of Widmanstätten ferrite plates from allotriomorph ferrite and the distributions of carbon concentration for the strength of anisotropy $\xi=0.6$ are shown in Fig. 6, where we use the wave numbers $N_{1}=5$, $N_{2}=8$ and $N_{3}=11$ for the perturbation of the initial interface of allotriomorph ferrite, which provide the interface morphology with short wavelength.

For the strength of anisotropy $\xi=0.6$, interfacial energy around the plate varies in the range from $0.94 \mathrm{~J} / \mathrm{m}^{2}$ at the plate tip to $0.42 \mathrm{~J} / \mathrm{m}^{2}$ at the plate side, the values of which correspond to the interfacial energy of incoherent and semicoherent interfaces, respectively. ${ }^{23)}$ Due to the high anisotropy of interfacial energy, in the early stage of growth $(2-20 \mu \mathrm{s})$, small sharp tips of the plate are formed directly on the allotriomorph ferrite, so that the total interfacial energy of the microstructure decreases. With further growth, it is observed that competitive growth and coalescence of neighboring plate tips occur simultaneously $(20-400 \mu \mathrm{s})$. In the later stage of growth $(400-1000 \mu \mathrm{s})$, the steady-state growth of Widmanstätten ferrite plates is observed.

We should note that the plate tips formed at the apex of the convex part of the initial allrotiomorph ferrite preferentially grow and their morphology easily changes to the platelike shape. This results also prove that the Widmanstätten ferrite plates are selectively developed because of the morphological instability, as suggested in the previous studies. ${ }^{26,28)}$

As the transformation proceeds, the increase of thickness is suppressed because of large carbon buildup at the plate side, which leads to a small driving force for the transformation. Therefore, the austenite phase existing between Widmanstätten ferrite plates cannot transform and remains as residual austenite.

Figures 7 and 8 show the microstructure evolutions and distributions of carbon concentration for $\xi=0.4$ and 0.3 , respectively. Upon comparison with the result shown in 

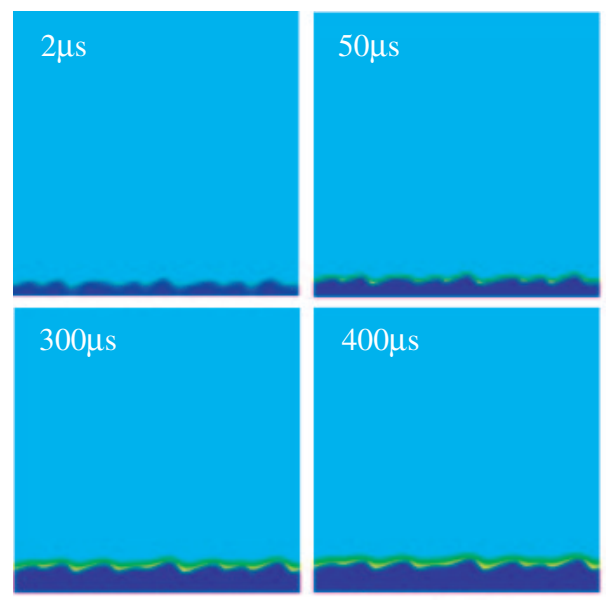

$\begin{array}{cccc}0.0014 & 0.0351 & 0.0688 & 0.1025 \\ \text { Carbon concentration } u_{\mathrm{c}}\end{array}$

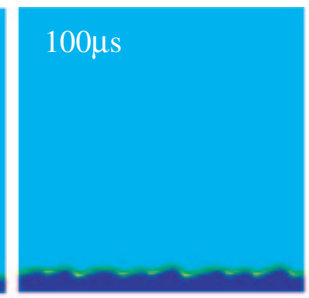

$600 \mu \mathrm{s}$

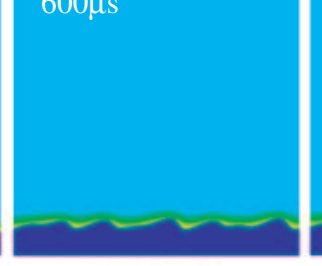

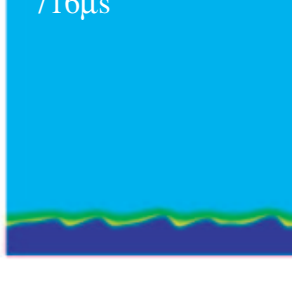

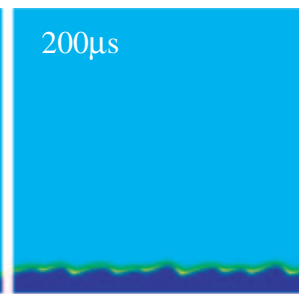

$716 \mu \mathrm{s}$

Fig. 8 Growth of Widmanstätten ferrite plates for $\xi=0.3$.
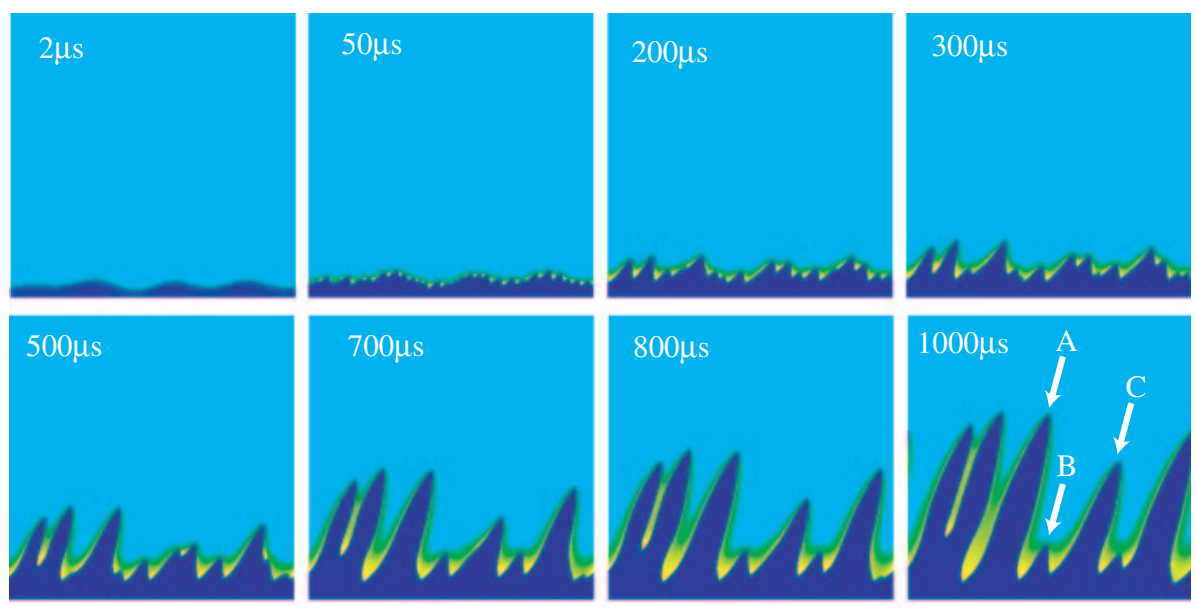

$0.0014 \quad 0.0306 \quad 0.0688 \quad 0.1025$
Carbon concentration $u_{\mathrm{c}}$

Fig. 9 Growth of Widmanstätten ferrite plates from allotriomorph ferrite with long wavelength initial interface for strength anisotropy $\xi=0.6$.

Fig. 6, we find that the formation of Widmanstätten ferrite plates is not observed with low strength of anisotropy, whereas the plates grow fast in the thickness direction. In the case of the lowest strength of anisotropy, $\xi=0.3$, there is continuous growth of allotriomorph ferrite instead of the formation of Widmanstätten ferrite plates. These simulation results clarify that high interface anisotropy is required for the formation of Widmanstätten ferrite plates from allotriomorph ferrite.

\subsubsection{Effects of initial shape of allotriomorph ferrite}

The morphological change from allotriomorph ferrite to Widmanstätten ferrite for the strength of anisotropy $\xi=0.6$ is shown in Fig. 9. In this case, the initial interface of allotriomorph ferrite is disturbed with the wave numbers $N_{1}=2, N_{2}=4$ and $N_{3}=3$, which produce longer wavelength of the interface than in the case described in section 4.2.1.

The simulation results show that the distribution of the

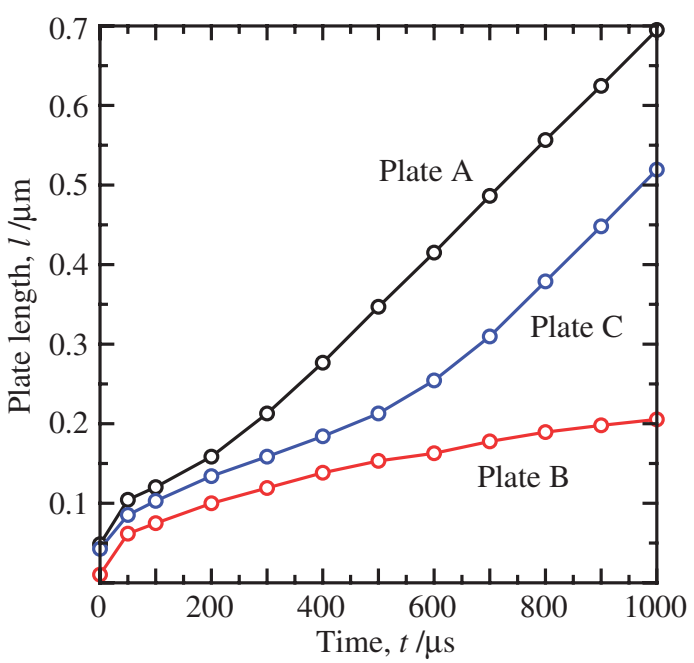

Fig. 10 Variation of lengths of the Widmanstätten ferrite plates indicated by white arrows in Fig. 9. 
Widmanstätten ferrite plates is clearly different from the results shown in Fig. 6, depending on the initial shape of allotriomorph ferrite. In particular, the number of Widmanstätten ferrite plates decreases with the decrease of the convex part of allotriomorph ferrite, while the thickness of the plates increases.

On the other hand, the growth kinetics of Widmanstätten ferrite is similar to the result shown in Fig. 6, even when the initial interface is disturbed by longer wavelengths. Figure 10 shows the variation of the lengths of the plates A, $\mathrm{B}$ and $\mathrm{C}$ indicated by arrows in Fig. 9. The tips formed at the convex part of the initial interface preferentially grow into Widmanstätten ferrite plates $\mathrm{A}$ and $\mathrm{C}$ via coalescence and competitive growth of the tips. The plate tip indicated by $\mathrm{B}$ can be formed even at the bottom of a wave, but the growth is greatly suppressed due to soft-impingement of the carbon diffusion field.

From the above results, we can conclude that the morphology, i.e., the plate spacing, plate thickness and location of the formation, of Widmanstätten ferrite plates strongly depends on the initial morphology of allotriomorph ferrite. These phase-field simulations clarify that Widmanstätten ferrite plates grow continuously from allotriomorph ferrite and that the morphological change of Widmanstatten ferrite can be described in terms of a diffusional growth mechanism, e.g., the ledge mechanism ${ }^{25,26)}$ or the instability theory. ${ }^{28-30)}$

\section{Conclusions}

The phase-field simulations for the growth of Widmanstätten ferrite plates in $\mathrm{Fe}-\mathrm{C}$ alloy were performed. We investigated the effects of the interface anisotropy on the growth kinetics of Widmanstätten ferrite plates by using the regularized gradient energy coefficient method which can introduce a wide range of interface anisotropy. It should be emphasized that a very sharp tip of Widmanstätten ferrite plates can be excellently simulated by employing the regularized gradient energy coefficient. Therefore, the morphology of Widmanstätten ferrite plate simulated in the present study is more realistic than that of previous numerical studies and is in good agreement with the results of experimental observation.

The simulation results showed that the lengthening rate of Widmanstätten ferrite plates increases with the increase of strength of anisotropy, which corresponds to the increase of interfacial energy at the plate tip. It was additionally noted that the morphology of plates becomes sharper upon increasing strength of anisotropy.

In order to study the effects of the interface anisotropy and the initial morphology of allotriomorph ferrite, the morphological change from grain boundary allotriomorph ferrite to Widmanstätten ferrite plates was simulated with a fluctuating initial interface. The simulation results revealed that the formation of Widmanstätten ferrite plates requires high anisotropy of interfacial energy. For the growth process, it was shown that Widmanstätten ferrite plates preferentially develop, because of the morphological instability, from the plate tips formed at the convex part of allotriomorph ferrite. Furthermore, it was clarified that the wavelength and the height of the disturbed interface of allotriomorph ferrite characterize the morphology of Widmanstätten ferrite plates.

In this study, we carried out the phase-field simulation of the formation of Widmanstätten ferrite plates and could simulate the very realistic morphology of Widmanstätten plate. As a future task, in order to investigate the growth process of Widmanstatten ferrite in the realistic scale such as that observed experimentally, we will adopt the thin-interface limit ${ }^{31)}$ which will enable us to use a larger interface thickness and to perform simulations in the extended computational domain with eliminating the nonphysically finite interface thickness effects.

\section{REFERENCES}

1) K. R. Kinsman, E. Eichen and H. I. Aaronson: Metall. Trans. A 6A (1975) 303-317.

2) H. I. Aaronson: Metall. Trans. A 24A (1993) 241-276.

3) A. Ali and H. K. D. H. Bhadeshia: Mater. Sci. Technol. 6 (1990) 781784.

4) Y. Ohmori, H. Ohtsubo, Y. C. Jung, S. Okaguchi and H. Ohtani: Metall. Mater. Trans. A 25A (1994) 1981-1989.

5) P. G. Ivantsov: Dokl. Akad. Nauk SSSR 58 (1947) 567.

6) M. Hillert: Jernkontorets., Ann. 141 (1957) 757-789.

7) R. Trivedi: Metall. Trans. 1 (1970) 921-927.

8) G. Caginalp: Arch. Rat. Mech. Anal. 92 (1986) 205-245.

9) R. Kobayashi: Phys. D. 63 (1993) 410-423.

10) J. A. Warren and W. J. Boettinger: Acta Metall. Mater. 43 (1995) 689703.

11) R. Kobayashi, J. A. Warren and W. C. Carter: Phys. D 140 (2000) 141150.

12) Y. Wang and A. G. Khachaturyan: Acta Mater. 45 (1997) 759-773.

13) I. Loginova, J. Odqvist, G. Amberg and J. Ågren: Acta Mater. 51 (2003) 1327-1339.

14) I. Loginova, J. Ågren and G. Amberg: Acta Mater. 52 (2004) 40554067.

15) T. Takaki, T. Fukuoka and Y. Tomita: J. Crys. Growth 283 (2005) 263271.

16) S. M. Allen and J. W. Cahn: Acta Metall. 27 (1979) 1085-1095.

17) M. Hillert: Metall. Trans. A 6A (1975) 5-19.

18) J. W. Cahn and J. E. Hilliard: J. Chem. Phys. 28 (1985) 258-267.

19) J.-O. Andersson and J. Ågren: J. Appl. Phys. 72 (1992) 1350-1355.

20) J. Ågren: Acta Metall. 30 (1982) 841-851.

21) J. Ågren: Scripta Metall. 20 (1986) 1507-1510.

22) J. J. Eggleston, G. B. McFadden and P. W. Voorhees: Phys. D 150 (2001) 91-103.

23) T. Furuhara: Testu-to-Hagane 89 (2003) 497-509.

24) M. G. Hall and H. I. Aaronson: Metall. Mater. Trans. A 25A (1994) 1923-1931.

25) M. Enomoto: Metall. Trans. A 22A (1991) 1235-1245.

26) M. Enomoto: Metall. Mater. Trans. A 25A (1994) 1947-1955.

27) C.-J. Huang, D. J. Browne and S. McFadden: Acta Mater. 54 (2006) $11-21$

28) H. I. Aaronson, D. Eylon, C. M. Cooke, M. Enomoto and F. H. Froes: Scripta Metall. 22A (1991) 435-440.

29) W. W. Mullins and R. F. Sekerka: J. Appl. Phys. 34 (1963) 323-329.

30) R. D. Townsend and J. S. Kirkaldy: Trans. ASM. 61 (1968) 605-619.

31) S. G. Kim, W. T. Kim and T. Suzuki: Phy. Rev. E 60 (1999) 71867197. 